\title{
小分子氢过硫化物供体的研究进展
}

\author{
许亚文 ${ }^{a}$ 王健鲲 ${ }^{b}$ 甄 乐*,b 王广基*,a,b \\ ( ${ }^{a}$ 广东药科大学药学院 广州 510006) \\ ( $b$ 中国药科大学药物代谢动力学重点实验室 南京 210009)
}

\begin{abstract}
摘要 小分子氢过硫化物(RSSH)具有独特的化学性质, 近年来其生物学活性备受瞩目. 近五年间, 生理或病理条件下 即可实现 RSSH 原位释放供体化合物取得了突破性的进展，这类供体将成为研究 RSSH 生理功能、作用机制以及治疗 潜能的有力工具. 按照激活模式将现有供体分子分为五类, 即酯酶激活型、ROS(活性氧物种)激活型、 $\mathrm{pH}$ 依赖型、光 激活型以及硝基还原酶激活型，并对各类供体进行综述. 现有供体在细胞水平表现出理想的抗氧化应激活性，部分供 体已经应用于心肌缺血再灌注模型的干预中, 并取得了积极效果. 此外, 还介绍了供体的释放机理及其概念验证过程, 并对供体分子的发展前景进行了展望.
\end{abstract}

关键词 氢过硫化物；供体；氧化应激；酯酶；硫烷硫

\section{Research Progress of Small-Molecular Hydropersulfide Donors}

\author{
Xu, Yawen ${ }^{a} \quad$ Wang, Jiankun ${ }^{b} \quad$ Zhen, Le ${ }^{*, b} \quad$ Wang, Guangji ${ }^{*, a, b}$
}

( ${ }^{a}$ College of Pharmacy, Guangdong Pharmaceutical University, Guangzhou 51000)

( ${ }^{b}$ Key Laboratory of Drug Metabolism and Pharmacokinetics, China Pharmaceutical University, Nanjing 210009)

\begin{abstract}
The unique chemical structure endowed small-molecular hydropersulfides (RSSH) with unclear but fascinating biological activities, which have been attracted much attention. The donors that could release RSSH smoothly in situ under physiological or pathological conditions will become powerful chemical tools for exploring the physiological functions, mechanism of action, and potential therapeutic benefit of RSSH. Over the past five years, a substantial breakthrough had been achieved in the design and development of RSSH donors, which is summarized in this perspective. According to the activation patterns, the existing donors are divided into five categories, namely esterase-activated donor, ROS (reactive oxygen species)-activated donor, $\mathrm{pH}$-dependent donor, light-activated donor, and nitroreductase-activated donor. All kinds of donors played ideal roles in improving the oxidative stress at the cellular level, part of which also show a positive therapeutic effect in the context of myocardial ischemia-reperfusion injury. Besides, the related release mechanisms and the proof of concept of the donors are briefly introduced. In the next five years, waves of novel donors are emerging. On one hand, diverse donors, with stable and controllable release rate, clear release process, and identified release components, will be designed. On the other hand, the indispensable toolbox of donors will be fully assembled for more in-depth and comprehensive biological activity researches.
\end{abstract}

Keywords hydropersulfides; donor; oxidative stress; esterase; sulfane sulfur

氢过硫化物(hydropersulfides) 是哺乳动物体内普遍 存在的一类活性硫物种(reactive sulfur species) ${ }^{[1]}$, 其结 构中含有两个共价结合的硫烷硫(sulfane sulfur) ${ }^{[2]}$; 而该 类硫原子处于 0 价或 -1 价, 具有较高的反应活性(图 $1)^{[3]}$. 硫醇仅具有亲核性, 而与之不同的是, 氢过硫化 物兼具亲核性及亲电性 ${ }^{[4]}$. 氢过硫化物具有较低的 $\mathrm{p} K_{\mathrm{a}}$
值(比硫醇 $\mathrm{p} K_{\mathrm{a}}$ 值低 $1 \sim 2$ ), 在生理环境中往往以负离子 形式存在，更易与亲电性物质发生反应. 而非离子形式 的氢过硫化物则具有亲电性, 能与弱亲核试剂如 $\mathrm{CN}^{-}$、 膦及硫醇等发生反应. 此外, 氢过硫化物的还原性也强 于相应硫醇, 其负离子形式可作为优良的单电子还原 剂，而被氧化所形成的氢过硫化物自由基具有良好的稳

\footnotetext{
* Corresponding authors. E-mail: i_m_zhenle@163.com; guangjiwang@hotmail.com Received January 5, 2021; revised March 18, 2021; published online April 16, 2021.

Project supported by the National Natural Science Foundation of China (Nos. 81973186, 81903707), the National Major Scientific \& Technological Special Project for "Significant New Drugs Development" (No. 2019ZX09301-151), and the Jiangsu Provincial Funds for Excellent Young Scientists (No. BK20200081)

国家自然科学基金(Nos. 81973186, 81903707)、国家科技重大新药创制专项(No. 2019ZX09301-151)及江苏省优青(No. BK20200081)资助项目.
} 
定性 ${ }^{[5]}$. 正是由于氢过硫化物具有独特的化学性质, 其 生物学活性才愈发受到关注.

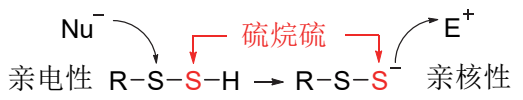

图 1 氢过硫化物的化学特性

Figure 1 Chemical properties of RSSH

人体内源性氢过硫化物可分为小分子氢过硫化物 (RSSH)和蛋白质氢过硫化物(PSSH). 常见的 RSSH 包括 半胱氨酸氢过硫化物 $(\mathrm{CysSSH})$ 、谷胱甘肽氢过硫化物 (GSSH)等. 在人与小鼠的大脑中, GSSH 浓度可达到 150 $\mu \mathrm{mol} / \mathrm{L}$, 在其他主要器官中其浓度约为 $50 \mu \mathrm{mol} / \mathrm{L}$; 而 CysSSH 在小鼠的不同组织中浓度约为 $1 \sim 4 \mu \mathrm{mol} / \mathrm{L}^{[6]}$. CysSSH 的内源性生成途径涉及三种酶: 胱硫醚- $\beta$-合成 酶(CBS)、胱硫醚- $\gamma$-裂解酶(CSE) 以及半胱氨酰-tRNA 合 成酶(CARS $)^{[7-8]}$. 体内半胱氨酸(Cys)经氧化作用生成胱 氨酸(CysSSCys), 并在 CBS/CES 作用下生成 CysSSH. 而两分子 Cys 能够在半胱氨酰-tRNA 合成酶(CARS)与 磷酸吡哆醛的作用下生成 $\mathrm{CysSSH}^{[8]}$. GSSH 的生成则可 能来自于 CysSSH 与谷胱甘肽 $(\mathrm{GSH})$ 之间发生的硫醇置 换反应. 此外, GSSH 可与谷胱甘肽二硫化物(GSSG)发 生反应得到多硫化物, 多硫化物又可在谷胱甘肽还原酶 作用下再次生成 GSSH ${ }^{[1,3,7]}$.

内源性 RSSH 的生物学意义目前尚不明晰, 其与 $\mathrm{H}_{2} \mathrm{~S}$ 的生理功能存在一定的交互与重叠. 一方面, RSSH 被认为是 $\mathrm{H}_{2} \mathrm{~S}$ 的前体, 许多 $\mathrm{H}_{2} \mathrm{~S}$ 产生的生理效应有可能 源于 $\mathrm{RSSH}^{[7,9]}$. 而另一方面, RSSH 也是线粒体硫氧化途 径中的重要中间体, 硫醌氧化还原酶能够以 GSH 作为 硫受体, 催化 $\mathrm{H}_{2} \mathrm{~S}$ 转化为 $\mathrm{GSSH}^{[10]}$. 此外, RSSH 还可以 直接参与细胞氧化还原稳态的调节 ${ }^{[1]}$. 例如, 8-硝基cGMP 是 NO/ROS 氧化还原信号通路中重要的第二信 使，而 RSSH 具有理想的还原性及亲核性，能够取代 8 位硝基, 并进一步生成 8-颈基-cGMP, 中止亲电性信号 传导 ${ }^{[12]}$.

越来越多的研究表明, 蛋白质过硫化在调节蛋白质 功能中起到重要作用. Cys 残基的翻译后修饰(如 $S$-过硫 化)被认为是生成 PSSH 的主要途径. 而近期研究表明, CysSSH 可借助 CARS 生成 $S$-过硫化半胱氨酰-tRNA, 并 直接参与翻译过程, 表达过硫化的蛋白质 ${ }^{[8]}$. 聚焦近三 年小分子氢过硫化物供体的研究进展进行综述, 有关蛋 白质氢过硫化物已有许多优秀综述与文献, 在此不再赘 述 ${ }^{[13]}$.

\section{1 小分子氢过硫化物供体研究进展}

RSSH 的生物活性及机制研究方兴未艾, 如何提供 稳定可控的 RSSH 分子亦成为领域热点. 内源性 RSSH
不易分离纯化，更加难以储存及递送; 而可分离纯化的 RSSH 往往含有大位阻烷基取代, 水溶性差且稳定性不 理想 ${ }^{[14]}$. 双烷基三硫化物(DATS)可作为 RSSH 供体，但 其产生的含硫物种十分复杂 ${ }^{[15]}$. 因此, 研究 RSSH 生物 学功能的一大障碍就是缺乏化学工具分子, 而设计生理 或病理条件下即可实现原位释放的 RSSH 供体成为解决 这一问题的关键.

\section{1 酯酶激活型氢过硫化物供体}

理想的酯酶激活型 RSSH 供体应在生理条件下保持 稳定，而在特定酯酶作用下释放 RSSH. 2017 年，王炳和 团队 [16]借助半缩醛的解离设计了供体 1 和 2 (图 2). 供体 1 或 2 在猪肝脂酶(PLE)的催化下生成关键中间体 $\mathbf{A} ; \mathbf{A}$ 自发解离并释放出一分子过硫化物 B 及一分子醛. 而在 释放体系中加入 $N$-乙酰半胱氨酸(NAC) 能够将 $\mathbf{B}$ 衍生 化为二硫化物 $\mathbf{C}$, 并释放一分子 $\mathrm{H}_{2} \mathrm{~S}$. 该研究通过 HPLC 对产物 $\mathbf{C}$ 及醛类化合物进行定性及定量分析，并用亚甲 基蓝法检测 $\mathrm{H}_{2} \mathrm{~S}$ ，揭示了供体的释放动力学. 实验表明, 供体能够有效地释放 RSSH, $T_{1 / 2}$ 仅为 12 至 $145 \mathrm{~s}$. 随后, 研究者借助 2,4-二硝基氟苯(FDNB)捕获过硫化产物 $\mathbf{B}$, 实现了对 $\mathbf{B}$ 的直接定量.

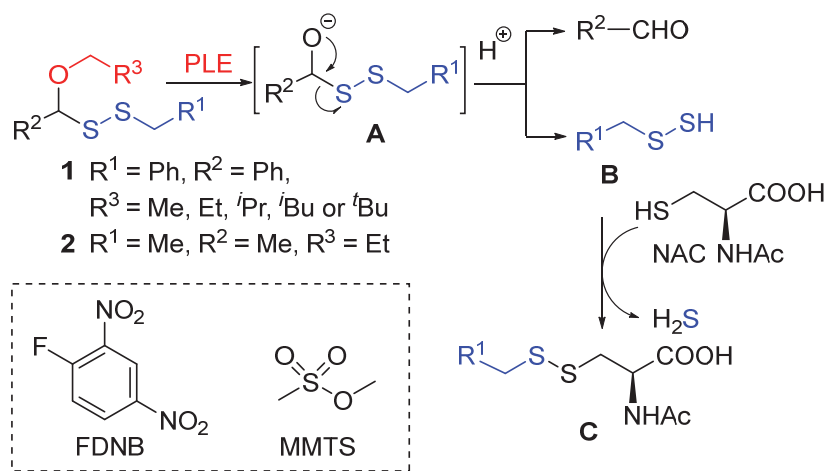

图 2 供体 $\mathbf{1}$ 与 $\mathbf{2}$ 的释放机制及捕获剂结构

Figure 2 Donors 1 and 2, their mechanisms of release, and the trapping agents

甲烷磺酸甲酯(MMTS) 曾被认为是一种 RSH 选择性 封闭试剂; 由于前期 RSSH 的生成体系较为复杂, 导致 MMTS 是否可衍生化 RSSH 一直存在争议. 而王炳和等 借助供体 1 证明了 MMTS 能够在生理条件下对 RSSH 进行烷基化.

供体 2 水溶性较好且副产物较低, 其作为候选化合 物在心肌缺血再灌注模型(MI/R)中表现出了保护作用. 实验通过闭塞左冠状动脉 (LCA)诱导小鼠心肌缺血; 缺 血 $45 \mathrm{~min}$ 后实施再灌注，供体 2 伴随再灌注给药; 再灌 注 $24 \mathrm{~h}$ 后再次闭塞 LCA, 并用 Evens Blue 及三苯基四 唑氯化物(TTC)对心肌双染，以评价 2 对危险区(AAR) 及梗死面积(INF)的影响. 研究结果表明, 供体干预组较 
对照组的梗死风险(INF/AAR)显著减低. 此外, 通过比 对急性心肌梗死标志物肌钲蛋白 I (cardiac troponin I) 的 浓度同样验证了 2 对 $\mathrm{MI} / \mathrm{R}$ 的保护作用.

GSSH 是生理条件下含量最丰富的小分子 RSSH. 2018 年, 王炳和课题组 ${ }^{[17]}$ 开发了首个 GSSH 供体 3 (BW-GP-401, 图 3). 3 在 PLE 的水解作用下释放出游离 酚羟基，并借助偕二甲基产生的 Thorpe-Ingold 效应实 现分子内环化, 释放 GSSH 的同时生成内酯副产物. 研 究者对内酯产物进行 HPLC 定量以预测 GSSH 的释放. 研究表明, 供体在无 PLE 的磷酸盐缓冲液(PBS)中保持 稳定; 而加入 PLE 后内酯的释放量在 $30 \mathrm{~min}$ 内可达到 $80 \%$. 随后的 FDNB 捕获实验中, 研究者发现 GSSH 的 衍生化产率并不高, 这是由于 GSSH 释放的同时也伴随 着自身歧化以及与硫醇 (如 NAC) 的偶联, 最终导致 $\mathrm{H}_{2} \mathrm{~S}$ 、多硫副产物 GSSSG 及 GSSNAC 的产生. 对释放 过程中产生的 $\mathrm{H}_{2} \mathrm{~S}$ 进行定量后发现, PLE 是 $\mathrm{H}_{2} \mathrm{~S}$ 释放的 必要条件, 而增加 NAC 有利于 $\mathrm{H}_{2} \mathrm{~S}$ 更快速的释放.

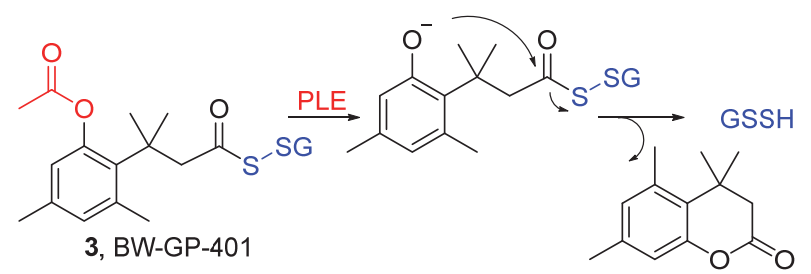

图 3 供体 3 及其释放机制

Figure 3 Donor 3 and its mechanism of release

甘油酫-3-磷酸脱氢酶(GAPDH) 是经典的糖酵解酶, 包含三个 Cys 残基, 分别为 C152、C156 及 $\mathrm{C} 247$, 其中 $\mathrm{C} 152$ 被认为是保持酶催化活性的必需位点. C152 残基 的氧化、亚硝化或过硫化均会导致酶的失活; 而抗氧化 剂及二硫键还原酶则促进酶恢复活性 ${ }^{[18]}$. 为此, 王炳和 等探究了 3 对 GAPDH 活性的影响. 研究表明, 3 对 GAPDH 的抑制作用呈剂量依赖性，且在二硫键还原试 剂 DTT 的作用下减弱, 因此可以推测 GSSH 通过 Cys 残基的过硫化作用抑制 GAPDH 的活性. 在 $\mathrm{H}_{2} \mathrm{O}_{2}$ 诱导的 H9C2 心肌细胞氧化应激模型中, $3(50 \mu \mathrm{mol} / \mathrm{Lol} / \mathrm{L})$ 所产 生的细胞保护作用要显著优于 $\mathrm{Na}_{2} \mathrm{~S}(150 \mu \mathrm{mol} / \mathrm{L})$ 和 $\mathrm{GSH}(150 \mu \mathrm{mol} / \mathrm{L})$ ，且呈浓度依赖性.

延长 RSSH 释放的半衰期对慢性炎症有潜在的积极 作用. 基于此, Matson 课题组 ${ }^{[19]}$ 于 2020 年设计了酯酶激 活型供体 $\mathbf{4}$ 以及与嵌段共聚物偶联而成的多聚供体 $\mathbf{5}$ (图 4), 探究了延长 RSSH 释放时间所产生的生物学活性.

4 被酯酶水解释放出酚氧负离子, 随后经 “ 1,6 -消 除” 释放出 $N$-乙酰半胱氨酸氢过硫化物 (NAC-SSH). 作 者以 PLE 激活供体, 并以 FDNB 作为捕获剂, 最终验证 了供体的释放. HPLC 分析结果显示, 4 在 PLE 作用下逐
渐分解，捕获产物 NAC-SS-DNB 逐渐生成. 供体 $\mathbf{5}$ 是将 供体 4 与 poly(HEMA-co-OEGMA)缀合而成, 其在 ${ }^{1} \mathrm{H}$ NMR 释放实验中的半衰期为 $36 \mathrm{~h}$, 远远大于供体 4 的释 放半衰期(1.6 h).

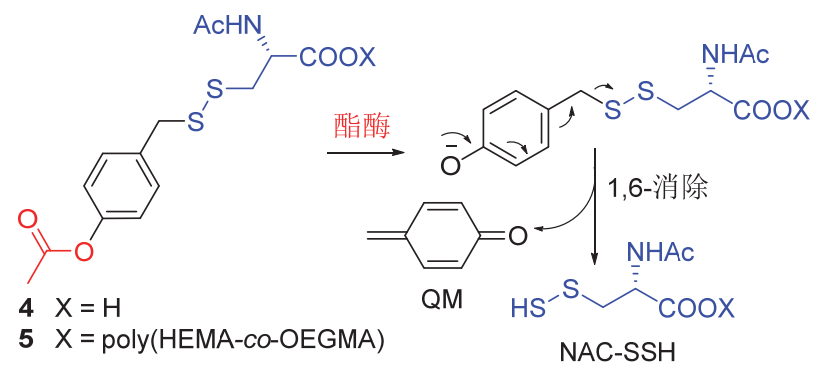

图 4 供体 4 与 5 及其释放机制

Figure 4 Donors 4 and 5, and their mechanisms of release

细胞活性实验中, 研究者首先考察供体是否对 $\mathrm{H}_{2} \mathrm{O}_{2}$ 刺激的 $\mathrm{H} 9 \mathrm{C} 2$ 心肌细胞具有保护作用, 结果显示给 药组细胞存活率有所改善, 且短效供体 4 药效优于长效 供体 5. 5-氟尿嘧啶(5FU)被认为是慢性的 ROS 诱导剂, 其刺激 $\mathrm{H} 9 \mathrm{C} 2$ 细胞 $4 \mathrm{~h}$ 后导致细胞存活率下降, 而 5 能 明显减少细胞毒性，且药效优于 4. 值得注意的是，两 供体均无法减缓 5FU 对 MCF-7 细胞所产生的毒性, 作 者推测 RSSH 供体有望与化疗药物联合用药, 在不影响 药效的基础上，降低化疗药对瘤外组织的毒副作用.

\subsection{ROS 激活型氢过硫化物供体}

2018 年, Matson 课题组 ${ }^{[20]}$ 开发了一类选择性响应 ROS 的 RSSH 供体 6 (BDP-NAC). 该供体结构中的芳基 硼酸频哪醇酯与 $\mathrm{HOO}^{-}$结合后发生 Baeyer-Villiger 重排, 生成的酚盐氧负离子经 1,6-消除释放出 NAC-SSH(图 $5)^{[21]}$. 研究者通过液质联用(LCMS)及 ${ }^{1} \mathrm{H}$ NMR 发现, 6 


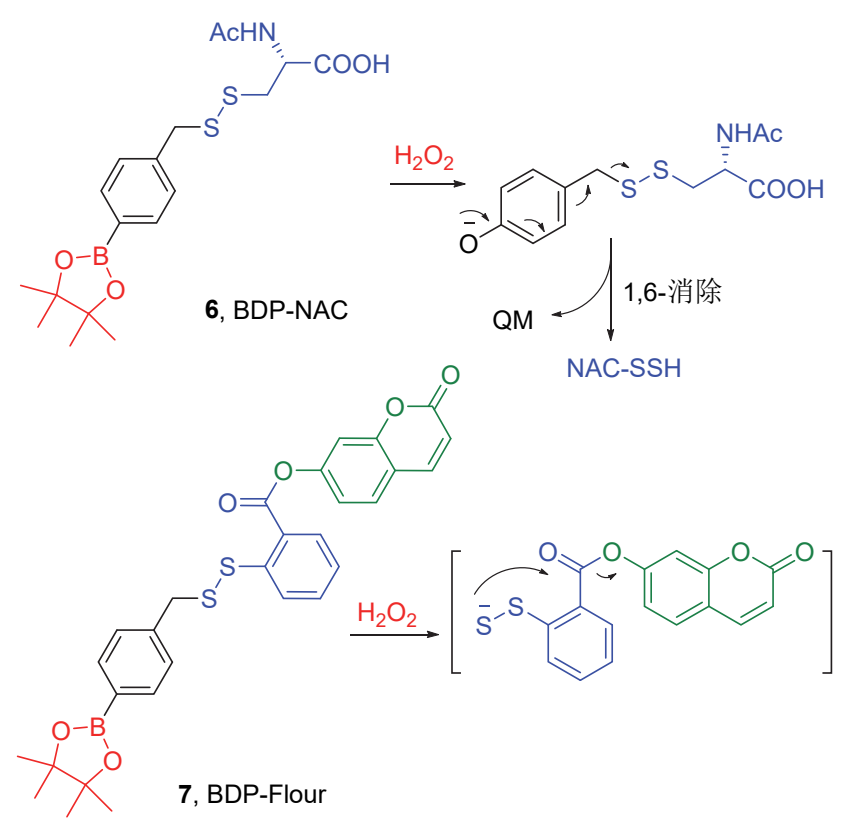

图 5 供体 6 与探针 7 及其释放机制

Figure 5 Donor 6 and probe 7, and their mechanisms of release 在 $\mathrm{H}_{2} \mathrm{O}_{2}$ 作用下逐渐降解, 并伴随 NAC-SSH 及对羟基苯 甲醇的生成. 此外，作者还设计并合成了苂光探针 7 (BDP-Flour). 7 被 $\mathrm{H}_{2} \mathrm{O}_{2}$ 激活后生成过硫化物中间体, 经 分子内环合释放 7-羟基香豆素(7-HCm). 通过比较荧光 强度的变化可以发现，该类苯硼酸酯供体能够被 $\mathrm{H}_{2} \mathrm{O}_{2}$ 选择性激活.

在 $\mathrm{H}_{2} \mathrm{O}_{2}$ 诱导的 $\mathrm{H} 9 \mathrm{C} 2$ 细胞氧化应激损伤模型中, $\mathrm{H}_{2} \mathrm{O}_{2}(100 \mu \mathrm{mol} / \mathrm{L})$ 导致细胞生存率降至约 30\%; 而 6 能 够发挥剂量依赖性的细胞保护作用, 给药 $200 \mu \mathrm{mol} / \mathrm{L}$ 后 细胞生存率提升至 100\%. 同样条件下，抗氧化剂 NAC、 $\mathrm{H}_{2} \mathrm{~S}$ 供体 $\mathrm{Na}_{2} \mathrm{~S}$ 及 $\mathrm{GYY} 4137$ 均未能表现出理想的保护作 用. 作者进一步猜测 NAC-SSH 比 $\mathrm{H}_{2} \mathrm{~S}$ 更能维持细胞的 氧化还原稳态. 该项工作提供了 RSSH 供体的全新模式, 为后续研究 RSSH 介导的生物学功能提供了设计思路.

考虑到上述 1,6-消除副产物亚甲基苯醌( $(\mathrm{QM})$ 的潜 在毒副作用 ${ }^{[22]}$, Chakrapani 课题组 ${ }^{[23]}$ 于 2018 年发展了一 类基于 1,4-消除的 RSSH 供体 8 . 该供体同样具有嗍酸 酯基团, 在释放 RSSH 的同时产生安全无毒的肉桂醛 (图 6).

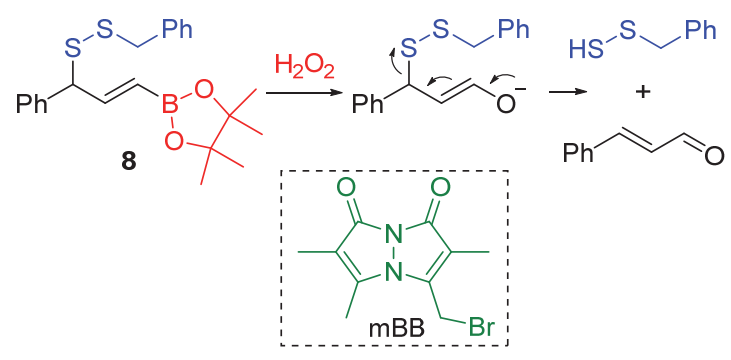

图 6 供体 8 及其释放机制

Figure 6 Donor $\mathbf{8}$, and its mechanism of release

在供体的释放研究中, 研究者将 8 与 10 equiv.的 $\mathrm{H}_{2} \mathrm{O}_{2}$ 共孵, 利用 HPLC 检测 8 的降解及肉桂醛的生成. 供体降解过程符合一级动力学, 速率常数 $k_{1}=5.3 \times 10^{-2}$ $\mathrm{min}^{-1}$; 而肉桂醛的释放速率常数与之接近 $\left(4.8 \times 10^{-2}\right.$ $\mathrm{min}^{-1}$ ). 研究者随后分别选用 FDNB 及单澳二胺 $(\mathrm{mBB})$ 对生成的 RSSH 进行捕获，两者的捕获速率相当. 值得 注意的是，肉桂醛与 RSSH亦会发生 Michael 加成，对此 该研究指出, 细胞内的硫醇(如 GSH)会竞争性地与肉桂 醛结合, 进而保证足量的 RSSH 发挥生物学功能.

生物活性研究选用甲菜醌及胡桃醌衍生物(JCHD) 诱导 DLD-1 结肠癌细胞产生氧化应激, 细胞存活率降 低; 而同时给予供体化合物后，细胞存活率显著提升， 且呈现剂量依赖性, 这说明 RSSH 的释放能有效的缓解 细胞氧化应激状态.

2020 年, Lukesh 等 ${ }^{[24]}$ 再次选择苯硼酸酯作为 ROS 特异性触发基团，并借助 “trimethyl lock” 前药策略，设 计了一类新型 RSSH 供体 9 (RAH393)，其释放过程与供 体 3 类似(图 7). 为了验证这一设计的合理性, 研究者将 供体离去基团替换为 7-HCm 与试卤灵(Resorufin), 合成 了荧光探针 10 (RAH225)及 11 (RAH2115), 两类探针的 激活均呈现较好的 $\mathrm{H}_{2} \mathrm{O}_{2}$ 特异性. 释放实验在考察了相 对荧光强度后发现, 50 equiv. $\mathrm{H}_{2} \mathrm{O}_{2}$ 能够驱动 10 在 $1 \mathrm{~h}$ 内 逐步释放出 7-HCm. 该研究还通过阐明 10 及其类似物 的构效关系，证明了嗍酸酯与偕甲基对苂光基团的释放 至关重要. 由于 11 具有较长的激发/发射波长, 因此应 用于指示百草枯所导致的 Hela 细胞内源性 $\mathrm{H}_{2} \mathrm{O}_{2}$ 累积. 此外，研究者借鉴探针 7 的结构，设计了含有二硫键的 苂光探针 12, 通过测定苂光强度随时间的变化, 计算出 探针的释放半衰期为 $60.5 \mathrm{~min}$, 表观速率常数为 $1.2 \times$ $10^{-2} \mathrm{~min}^{-1}$; 此外，作者还通过质谱学手段验证了环状 二硫化物的生成. 

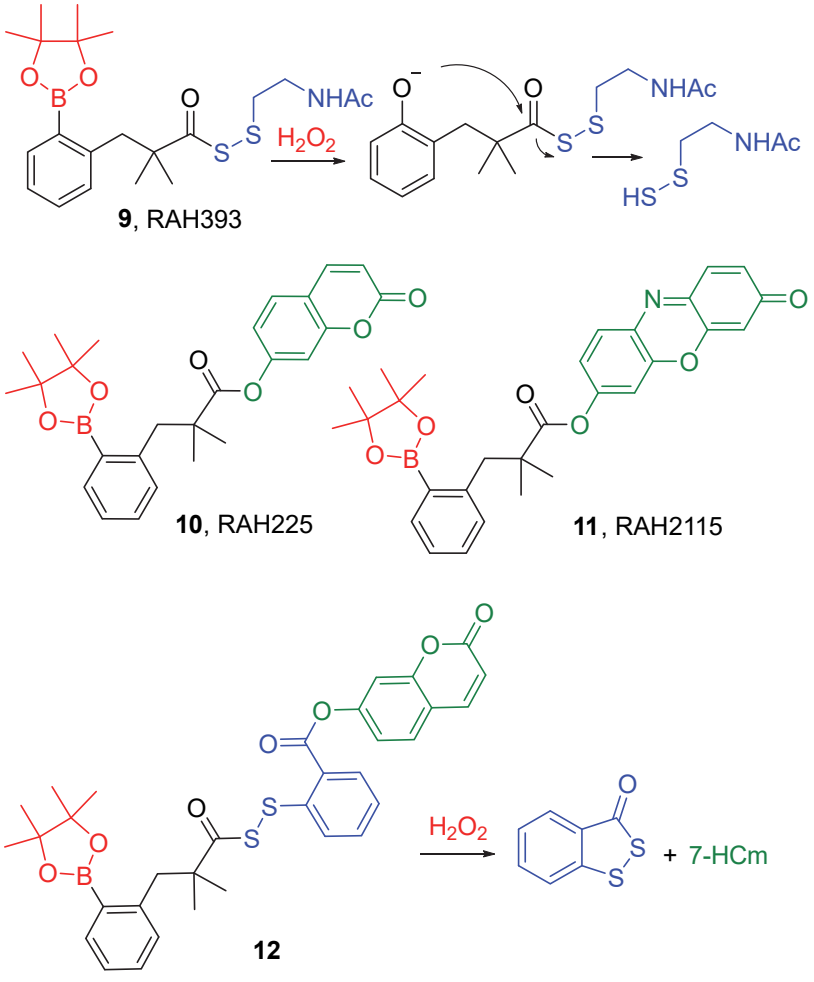

图 7 供体 9、探针 10 12 及其释放机制

Figure 7 Donor 9, probes 10 12 , and their mechanisms of release

基于荧光探针的释放验证实验，作者利用捕获剂 FDNB 对 $N$-乙酰基半胱胺进行衍生化, 并通过 LCMS 对 其定量, 进一步阐明了 9 的释放. $9(200 \mu \mathrm{mol} / \mathrm{L})$ 在 $\mathrm{H}_{2} \mathrm{O}_{2}$ $(1 \mathrm{mmol} / \mathrm{L})$ 中存在稳定释放, 反应 $15 \mathrm{~min}$ 后生成量约为 $62.9 \mu \mathrm{mol} / \mathrm{L}$ (产率 $31.4 \%$ ). 而不含嗍酸酯结构的对照化 合物并未检测到释放 RSSH. 随后的细胞活性研究中, $\mathrm{H}_{2} \mathrm{O}_{2}(100 \mu \mathrm{mol} / \mathrm{L})$ 导致 HeLa 细胞存活率降至 33\%; 而 提前 $1 \mathrm{~h}$ 给予供体后, $\mathrm{H}_{2} \mathrm{O}_{2}$ 造成的细胞损伤明显降低, 细胞存活率接近 $100 \%$.

另外值得注意的是, 9 具有酰基过硫化物结构, 在 体内硫醇 RSH 作用下容易降解为硫代羧酸. 而采用亚 甲基蓝法研究发现, 9 在 RSH 存在下还表现为一类有效 的 $\mathrm{H}_{2} \mathrm{~S}$ 供体.

超氧离子(superoxide anion, SOA)作为一类 ROS, 主要由线粒体的呼吸作用产生. 而线粒体功能障碍往往 导致 SOA 内稳态被破坏, 造成细胞损伤 ${ }^{[25] .} 2020$ 年, Matson 课题组 ${ }^{[26]}$ 设计了一类 SOA 特异性响应的 RSSH 供体 13 (SOPD-NAC, 图 8). 该供体结构中的二苯基次 膦酸酯能够捕获 SOA 并水解出氧负离子, 后者经历 $1,6-$ 消除释放 NAC-SSH(参见供体 $4 \sim 6$ ).

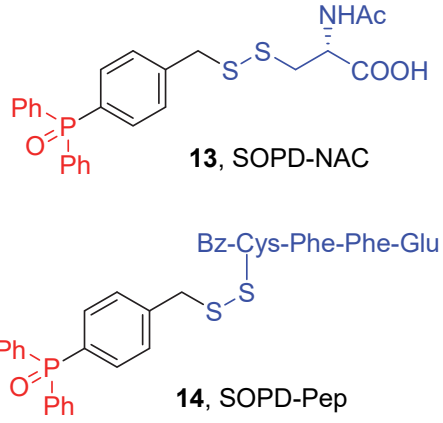

图 8 供体 13 与 14

Figure 8 Donor 13 and 14

自组装肽能够自发形成纳米结构或水凝胶，具有优 良的生物降解性和生物相容性. 鉴于 Matson 等已成功 将 $\mathrm{H}_{2} \mathrm{~S}$ 供体与自组装肽结合, 研究者将 SOPD 结构缀合 至自组装肽 (Bz-Cys-Phe-Phe-Glu) 得到短肽供体 14 (SOPD-Pep, 图 8), 以期改善水溶性及细胞靶向性. 该 短肽具有两亲性，透射电镜观察表明其能够自发形成纳 米带, 具有理想的组装性质.

生物活性研究中, 研究者采用丁硫氨酸-亚砜亚胺 耗竭 H9C2 细胞内 GSH 以提高 SOA 水平, 同时给予 $\mathrm{H}_{2} \mathrm{~S}$ 探针 WAP-5 以及供体 13 或 14. 结果显示, 14 干预组苂 光强度最高, 说明 SOA 促进了 RSSH 的释放. 而同样条 件下，将荧光探针更换为二氢乙锭，以指示胞内氧化应 激水平. 结果显示, 模型组 ROS 含量明显高于供体干预 组. 随后，作者选用佛波醇-12-十四酸酯-13-乙酸酯去 诱导 RAW 264.7 巨噬细胞产生炎性损伤，而造模后给予 供体能够显著改善细胞存活率. 此外, 供体对细胞的保 护作用要优于 $\mathrm{H}_{2} \mathrm{~S}$ 供体 GYY4137 及 $\mathrm{Na}_{2} \mathrm{~S}$.

\section{$1.3 \mathrm{pH}$ 依赖型氢过硫化物供体}

2013 年, Galardon 等 ${ }^{[27]}$ 发现乙酰二硫化物能够与胺 发生分子间乙酰基转移，生成乙酰基过硫化物 AcSSH (图 9, box). 在此基础上, 该课题 ${ }^{[28] 于 ~} 2014$ 年报道了 RSSH 供体 15. 在 Tris 缓冲液 $(\mathrm{pH}=7.4)$ 中, 供体 $\mathbf{1 5}$ 的氨 基盐酸盐逐渐游离，亲核性氨基借助偕二甲基效应自发 进行分子内加成一消除反应，实现 $N$-甲氧羰基转移并暴 露出过硫化基团. 该转移过程与 $\mathrm{pH}$ 值密切相关, 通过 对比供体在 $\mathrm{pH}$ 为 $8.5 、 7.4$ 及 6.5 时的表观速率常数发 现, 碱性越强, 释放速率越快; 而供体在酸性缓冲液 $(\mathrm{pH}=2.7)$ 中比较稳定, $120 \mathrm{~min}$ 内仅降解不到 $5 \%$. 此外, 供体 15 的 $N$-乙酰化衍生物无法游离出过硫化基团, 证 明甲氧羰基转移是供体发挥作用的关键，而并非依赖甲 氧羰基水解. 研究者使用 $N$-乙基马来酰亚胺(NEM)作为 捕获剂, 借助 LCMS 确证了捕获产物 RSS-NEM 的结构. 


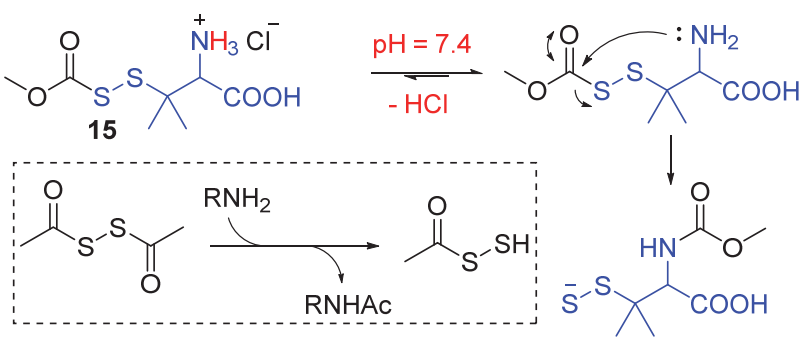

图 9 供体 $\mathbf{1 5}$ 及其释放机制

Figure 9 Donor 15 and its mechanism of release

氢过硫化物是一类亚稳定结构, 研究人员发现供体 15 释放过硫化基团后, 会发生自身二聚反应; 但值得注 意的是, 供体 15 浓度升高至 $100 \mathrm{mmol} / \mathrm{L}$ 后, 仍未检测 到 $\mathrm{H}_{2} \mathrm{~S}$ 的释放. 以上结果一方面排除了过硫化基团是自 身歧化所产生, 一方面说明 $\mathbf{1 5}$ 能够避免 $\mathrm{H}_{2} \mathrm{~S}$ 的干扰, 适 合于研究硫烷类化合物的生物学活性. 随后的活性研究 中, 15 能以较高收率将 8-硝基-cGMP 转化为 8-颈基cGMP. 此外, 15 可将三价铁细胞色素 $\mathrm{C}$ 定量转化为亚铁 细胞色素 $\mathrm{C}$, 而经典的硫醇如 GSH 或青霉胺则无法实现 这一过程, 可见 RSSH 是一类生物体内高效的单电子还 原剂.

2018 年, Xian 课题组 ${ }^{[29]}$ 开发了一系列含有 $O$-硅烷 基的 RSSH 供体 16, 其机理与供体 $\mathbf{1}$ 类似, 均是基于 1,2-O,S-消除机制释放(图 10). 由于酸性条件下硅醚容 易解离, 研究人员对不同 $\mathrm{pH}$ 下 RSSH 的释放速率进行 了考察. 研究表明, 供体释放过程呈现 $\mathrm{pH}$ 值依赖性, $\mathrm{pH}$ 值越低则释放速率越快; 含有三甲基硅基(TMS)的供体 在弱酸性条件 $(\mathrm{pH}=5.0 / 6.0)$ 下即可释放; 而在 $\mathrm{pH}=7.4$ 时释放速率变慢, 同时得到二硫化物或三硫化物的混合 物. 连接三乙基硅基(TES)的供体相对稳定, 在不同 $\mathrm{pH}$ 缓冲系统中均无明显释放.

此外, 研究者还考察了 16 在氟离子 $(\mathrm{KF})$ 存在下的 释放. 含 TES 的供体在氟离子存在下能够缓慢释放, 但 释放速率远远小于含 TMS 的供体.

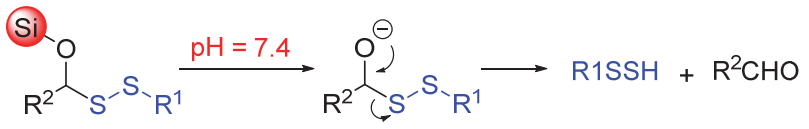

16

图 10 供体 16 及其释放机制

Figure 10 Donor 16 and its mechanism of release

Toscano 课题组 ${ }^{[30]}$ 于 2018 年报道了一类新型 RSSH 供体 17, 希望通过异硫䐂结构的脱除实现 RSSH 的释放 (图 11). 研究者选用 NEM 作为衍生化试剂, 通过 LCMS 及 ${ }^{1} \mathrm{H}$ NMR 对捕获产物 RSS-NEM 进行定量, 以研究 RSSH 的释放程度. 最初设计的化合物不含偕二甲基,
捕获实验中并没有检测到目标产物，而是生成了两类副 产物, 即三硫化物(RSSSR)与硫代亚磺酸酯(RS(O)-SR). 随后在供体结构中引入偕二甲基, RSS-NEM 的产率明 显增加. 在进一步构效关系探索中, 羧酸甲酯的引入虽 然减少了副产物 RS(O)SR 的生成，但未能增加 RSSH 的 释放. 而在异硫脲结构中引入 $N$-甲基后， RSS-NEM 的 产率下降，且仍伴随大量副产物 RS(O)SR 生成.

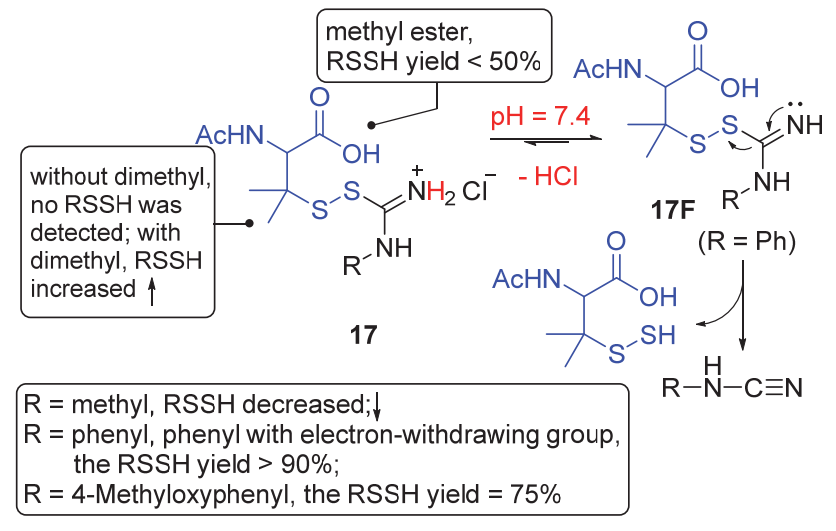

图 11 供体 17 及其释放机制

Figure 11 Donor 17 and its mechanism of release

当在异硫脲结构中引入 $N$-苯基后, RSS-NEM 的产 率显著提升，在反应体系中也未检测到 RS(O)SR. 随后， 研究者对苯环取代基进行考察, 并用核磁定量的方法阐 明了 RSSH 的产率及释放半衰期. 研究结果显示, 当苯 基含有吸电子基团时， RSSH 产率均能大于 $90 \%$, 释放 半衰期延长; 而当苯基对位含有甲氧基时，RSSH 产率 下降至 75\%，释放半衰期稍有缩短.

值得注意的是，供体释放过程并未检测到 $N$-苯基 腿, 而是有大量苯基氰胺生成, 其机理如图 11 所示. 异 硫脲盐在中性缓冲液中逐步转化为游离形式的供体 $17 F$ ，该中间体经历消除反应得到苯基氧胺及 RSSH. 当 苯基上含有给电子基团时, 17 在缓冲液中转化为 $17 \mathrm{~F}$ 的 程度降低，因而释放产率下降; 而苯基上含有吸电子基 团时，17F 亚胺孤对电子不易发生消除反应，因而半衰 期延长.

成盐氨基可在中性缓冲液中逐渐解离, 利用游离氨 基的亲核性是 $\mathrm{pH}$ 依赖型供体实现 RSSH 释放的关键. 2020 年, Toscano 课题组 ${ }^{[31]}$ 报道了具有硫代氨基甲酸酯 结构的 RSSH 供体 18(图 12). 非亲核性的铵盐在生理条 件下 $(\mathrm{pH}=7.4)$ 会产生游离氨基，后经分子内环化-消除 释放 RSSH. 

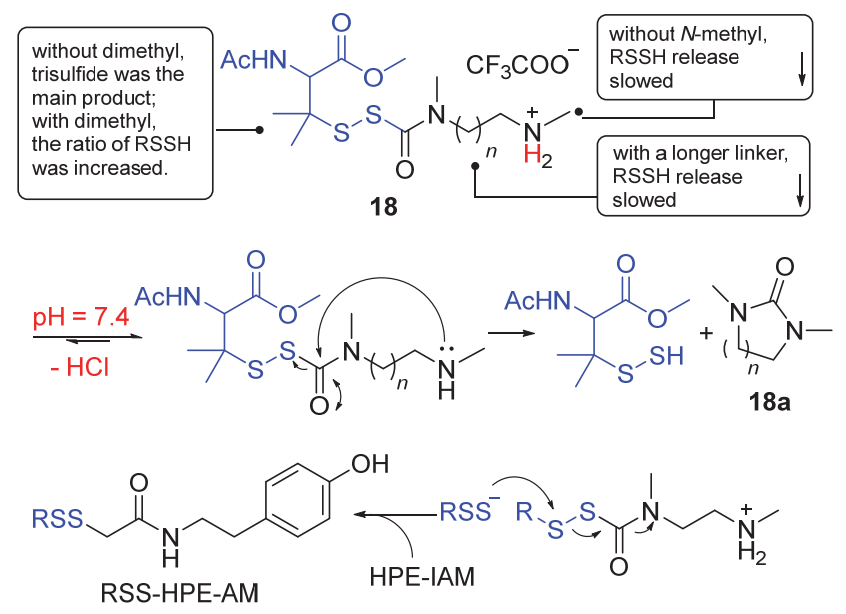

$\rightarrow$ RSSSR + COS

图 12 供体 18 及其释放机制

Figure 12 Donor 18 and its mechanism of release

研究者对供体 18 进行了构效关系研究. 当供体为 游离羧酸且无偕二甲基结构时, RSSH 以 NAC-SSH 形式 释放, 与 HPE-IAM 竞争性的进攻 18, 生成的三硫化物 RSSSR 为主要产物. 而供体为羧酸甲酯衍生物且含偕 二甲基结构时, RSSH 与 $\mathbf{1 8}$ 的反应性下降, RSS-HPE$\mathrm{AM}$ 成为主产物. 当去除末端 $N$-甲基后, 伯胺亲核能力 降低, 释放 RSSH 速率变小; 当两个氮原子之间的碳链 延长后, 分子内环化的难度增加, 释放 RSSH 速率会进 一步减缓. 含有羧酸甲酯的供体能够保证 RSSH 产率高 于 $90 \%$, 而释放半衰期可以通过结构修饰进行调节, 这 为后续供体的设计与相关生物学研究提供了重要参考.

此外，若将末端氨基去除，供体 $\mathbf{1 8}$ 无法释放 RSSH; 即使在释放实验中加入 5 equiv. 的正丁胺, 供体仍能稳 定存在. 值得注意的是, 当外加 NAC 后, 硫代氨基甲酸 酯结构并没有发生硫醇解, 而是产生了 $\mathrm{H}_{2} \mathrm{~S}$ 的前体物质 硫化碳(COS). COS 的产生是否会干扰 RSSH 的释放 呢? 作者通过 LCMS 检测伴生物 18a 后发现, 当供体释 放速率较快时, RSSH 释放并不受外加硫醇的干扰; 而 具有较长释放半衰期的供体, 其 RSSH 释放程度则明显 降低. 另外, 该研究还直接借助膜进样质谱仪(MIMS) 对生成的 COS 进行定量, 实验结果与上述一致.

在细胞保护作用的研究中, $\mathrm{H} 9 \mathrm{C} 2$ 细胞经供体预处 理 $2 \mathrm{~h}$ 后, 再进行 $\mathrm{H}_{2} \mathrm{O}_{2}$ 刺激 $2 \mathrm{~h}$, 与空白组相比, 给药组 能够明显提升细胞存活率. 在离体心脏 Langendorff 灌 流模型中，心脏缺血 $20 \mathrm{~min}$ 后进行 $90 \mathrm{~min}$ 再灌注，前 7 $\min$ 再灌注伴随给予 $\mathbf{1 8}$ 或 $\mathrm{KH}$ 缓冲液. 再灌注结束后使 用 TTC 染色发现, KH 缓冲液梗死面积为 $42 \%$, 而供体 给药后梗死面积减小至 $16 \% .18$ 表现出较好的抗氧化应 激作用，并能改善 $\mathrm{MI} / \mathrm{R}$.

\section{4 光激活型氢过硫化物供体}

光激活无需依赖机体内部化学环境的改变, 仅需借 助自身光敏基团即可实现供体小分子的释放. 邻硝基茮 基 $(\mathrm{ONB})$ 作为经典的光敏基团，不仅能够实现单光子激 发, 且具有一定的双光子激发特征; 其在快速释放的同 时不产生复杂副产物. 基于 ONB 的光敏特性, Singh 团 队) ${ }^{[32]}$ 于 2019 年开发了首个光激活型 RSSH 供体 19(图 13. ONB 结构吸收特定波长光子后，供体激发至三线态; 该状态下硝基发生抽氢反应形成 $a c i$-硝基; 后者环化为 苯并异噁唑中间体，随后五元环裂解为羰基及亚硝基， 并释放出离去基团 NAC-SSH.

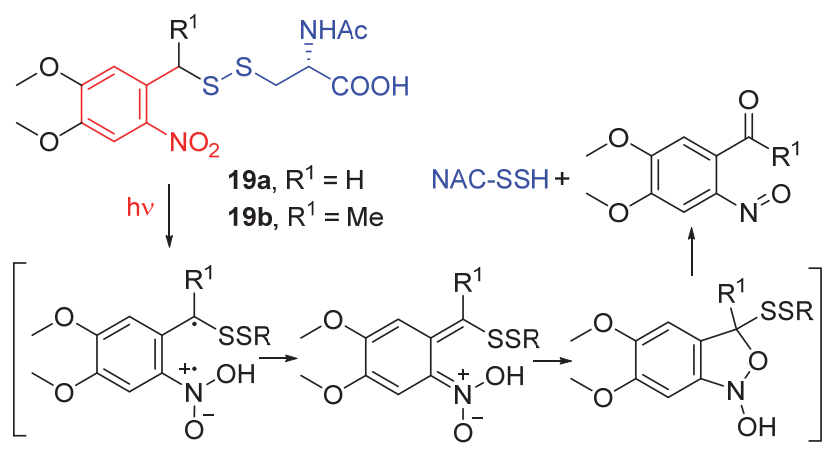

图 13 供体 19 及其释放机制

Figure 13 Donor 19 and its mechanism of release

研究者首先确定了供体 19a 与 $19 b$ 在含 $10 \%$ 胎牛血 清或 10 equiv.硫醇(NAC 或 GSH)的混合溶剂(乙腈/PBS, $V: V=3: 7, \mathrm{pH}=7.4)$ 中均具有良好的稳定性. 随后, 分别利用 HPLC 及 ${ }^{1} \mathrm{H}$ NMR 验证了供体在 $365 \mathrm{~nm}$ 单光 子激发下存在光解现象, 并借助 LCMS 捕获到了光解产 物. 供体 $19 \mathbf{a}$ 与 $19 \mathrm{~b}$ 的光解量子产率分别为 0.07 与 0.36 ; 而 19a 的双光子吸收截面约为 $0.033 \mathrm{GM}, 730 \mathrm{~nm}$ 双光子 激发下的光解速率常数为 $k_{\mathrm{obs}}=6.1 \times 10^{-4}$.

在 RSSH 捕获实验中, 利用 $\mathrm{mBB}$ 捕获不同时间点 释放的 NAC-SSH，随着照射时间的延长, $455 \mathrm{~nm}$ 特征性 荧光强度逐渐增加; 而以 FDNB 为捕获剂时, 通过对照 NAC-SS-DNB 标准品, 利用 HPLC 对 RSSH 的释放进行 定量. 与此同时, FDNB 捕获 NAC-SSH 的过程中给予明 暗交替刺激(ON-OFF), 实验结果再次确证只有光照才 能形成 NAC-SS-DNB.

细胞实验中, HeLa 细胞分别给予供体 19a、19b、 GSH、NAC、HCys 及 $\mathrm{Na}_{2} \mathrm{~S}$, 随后再进行 $\mathrm{H}_{2} \mathrm{O}_{2}$ 刺激. 结 果显示, 供体的细胞保护能力优于其它给药组. 此外, 二氯荧光黄双乙酸盐(DCFDA)活性氧染色实验同样验 证了上述结论.

\section{5 硝基还原酶激活型氢过硫化物供体}

越来越多的实验证据表明, 肠道微生物组 (gut mi- 
crobiome)与人类的健康及疾病息息相关 ${ }^{[33]}$. 为了探究 外源性活性硫物种, 尤其是 RSSH 对肠道微生物组的作 用, Matson 课题组 ${ }^{[34]}$ 于 2021 年设计并合成了可于肠道 内释放的新型供体 20 (NDP-NAC, 图 14). 该供体能够 借助哺乳动物肠道内细菌源硝基还原酶(nitroreductase, $\mathrm{NR}$ )实现特异性激活.

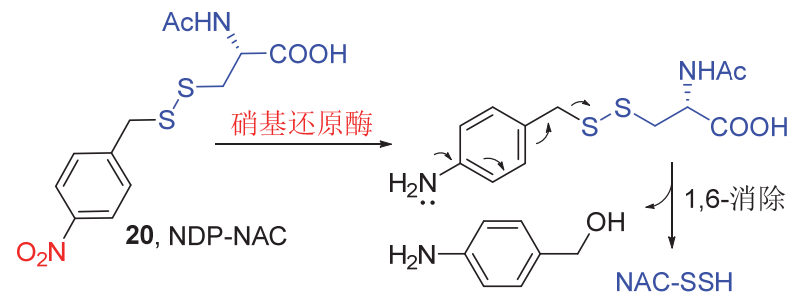

图 14 供体 20 及其释放机制

Figure 14 Donor 20 and its mechanism of release

在 NR 与 NADH 的共同作用下，供体结构中的硝基 被还原为含亚硝基或氨基结构的中间体，这类中间体随 即发生 1,6-消除, 释放 NAC-SSH 及副产物对氨基苯甲 醇. 作者首先选用 FDNB 为捕获剂, 在含有 NR(大肠杆 菌源)及 NADH 的 PBS 溶液 $(\mathrm{pH}=8.0)$ 中验证了 NAC$\mathrm{SSH}$ 的释放; 随后, 利用 ${ }^{1} \mathrm{H}$ NMR 考察了供体的降解动 力学以及 Cys 存在下的稳定性.

在生物活性研究中, 供体对 $\mathrm{H} 9 \mathrm{C} 2$ 细胞的安全性良 好, 也不影响大肠杆菌的活力, 但能够剂量依赖性的抑 制两类条件致病菌[李斯特菌 (L. monocytogenes) 及金黄 色葡萄球菌( $S$. aureus)]的增殖. 通过 MALDI-TOF 鉴定 小鼠粪便细菌后发现, 与 PBS 组相比, 给药组细菌种类 发生明显变化. 通过气相色谱对粪便样本中的短链及中 链脂肪酸分析可知, 给药组菌群变化诱导了庚酸含量的 大幅增加. 随后，根据细菌分类丰度进行样品聚类分析 可知, 与 PBS 组相比, 供体组菌群种类在 Phylum 水平 上存在显著差异, 其中狍子形成菌 T. sanguinis 存在大 量增殖, 而庆氧性条件致病菌 Synergistales 数量下降. 作者还采用鸟枪法宏基因组学(shotgun metagenomics) 预测微生物蛋白功能及其对机体的影响. 分析结果表 明, 给予供体后菌群的变化很有可能影响到机体遗传信 息的处理与翻译, 以及脂质代谢功能, 并与代谢性疾病 相关.

\section{2 总结与展望}

RSSH 是一类含有硫烷硫结构的活性分子, 兼具亲 核性、亲电性及还原性; 独特的化学结构赋予其多元的 生物学活性; 探究其生物功能及作用机制富有吸引力与 挑战性，近年来已逐渐成为领域内深入探索的热点. 近 三年间, 新型 RSSH 供体的发现如雨后春笋, 借助供体
已实现了单一组分 RSSH 的原位释放，这为后续研究其 生物学活性提供了关键策略. 现有研究围绕多样化的生 理或病理条件, 设计了多种供体激活模式; 根据激活模 式的不同，我们将目前所报道的供体分为五类，即酷酶 激活型、 $\mathrm{ROS}$ 激活型、 $\mathrm{pH}$ 依赖型、光激活型及硝基还 原酶激活型. 现有研究通过 NMR、HPLC、LC-MS 以及 苂光探针合成等手段验证了供体在生物缓冲体系内的 释放, 并在细胞水平明确了供体理想的抗氧化应激活 性; 此外, 部分供体(如供体 2 与 18)已经应用于心肌缺 血再灌注动物模型的干预，并取得了积极效果.

$\mathrm{RSSH}$ 供体与经典的气体信号分子(如 $\mathrm{NO} 、 \mathrm{H}_{2} \mathrm{~S}$ 、 $\mathrm{CO}$ 等)供体类似，其释放过程需要具备可控性，即供体 能够在合适的靶部位以适宜的速率实现 RSSH 的释放. 现有供体已在体外缓冲系统中验证了 RSSH 的释放; 而 对于其在细胞内甚至体内的释放过程还需要在未来的 研究中进一步阐明. 随着研究的深入, 新一代 RSSH 供 体将在特定内环境中具备稳定可控的释放速率、明确的 释放组分以及清晰的释放过程. 另一方面，现有供体大 部分表现出在细胞层面的抗氧化应激活性，这有助于揭 示供体的胞内作用机制; 而供体化合物在体内疾病模型 中是否同样具有干预作用，这也将成为今后研究的重 点.

总之，在未来的五年间，我们相信这一领域还会有 新的亮点交相绽放, RSSH 供体有潜力成为探究 RSSH 生物学功能及机制的必备分子，而设计结构新颖且体内 外释放机制明确的供体有望为生物活性研究提供更多 的选择.

\section{References}

[1] The representative reviews of hydrogen persulfides in recent years see:

(a) Saund, S. S.; Sosa, V.; Henriquez, S.; Nguyen, Q. N. N.; Bianco, C. L.; Soeda, S.; Millikin, R.; White, C.; Le, H.; Ono, K.; Tantillo, D. J.; Kumagai, Y.; Akaike, T.; Lin, J.; Fukuto, J. M. Arch. Biochem. Biophys. 2015, 588, 15.

(b) Kasamatsu, S.; Nishimura, A.; Morita, M.; Matsunaga, T.; Hamid, H. A.; Akaike, T. Molecules 2016, 21, 1721.

(c) Fukuto, J. M.; Ignarro, L. J.; Nagy, P.; Wink, D. A.; Kevil, C. G.; Feelisch, M.; Cortese-Krott, M. M.; Bianco, C. L.; Kumagai, Y.; Hobbs, A. J.; Lin, J.; Ida, T.; Akaike, T. FEBS Lett. 2018, 592, 2140 .

(d) Filipovic, M. R.; Zivanovic, J.; Alvarez, B.; Banerjee, R. Chem Rev. 2018, 118, 377 .

(e) Lau, N.; Pluth, M. D. Curr. Opin. Chem. Biol. 2019, 49, 1. (f) Sawa, T.; Motohashi, H.; Ihara, H.; Akaike, T. Biomolecules 2020, 10, 1245.

[2] (a) Iciek, M.; Bilska-Wilkosz, A.; Gorny, M. Acta Biochim. Pol. 2019, 66, 533.

(b) Shinkai, Y.; Kumagai, Y. Toxicol. Sci. 2019, 170, 3.

[3] (a) Ono, K.; Akaike, T.; Sawa, T.; Kumagai, Y.; Wink, D. A.; Tantillo, D. J.; Hobbs, A. J.; Nagy, P.; Xian, M.; Lin, J.; Fukuto, J. M. Free Radical Biol. Med. 2014, 77, 82.

(b) Park, C.-M.; Weerasinghe, L.; Day, J. J.; Fukuto, J. M.; Xian, M. Mol. Biosyst. 2015, 11, 1775. 
(c) Cuevasanta, E.; Moller, M. N.; Alvarez, B. Arch. Biochem. Biophys. 2017, 617, 9.

[4] (a) Everett, S. A.; Folkes, L. K.; Wardman, P.; Asmus, K. D. Free Radical Res. 1994, 20, 387.

(b) Cuevasanta, E.; Lange, M.; Bonanata, J.; Laura Coitino, E.; Ferrer-Sueta, G.; Filipovic, M. R.; Alvarez, B. J. Biol. Chem. 2015, 290, 26866.

(c) Benchoam, D.; Semelak, J. A.; Cuevasanta, E.; Mastrogiovanni, M.; Grassano, J. S.; Ferrer-Sueta, G.; Zeida, A.; Trujillo, M.; Moller, M. N.; Estrin, D. A.; Alvarez, B. J. Biol. Chem. 2020, 295, 15466.

[5] (a) Everett, S. A.; Wardman, P. Method Enzymol. 1995, 251, 55. (b) Jin, F.; Asatryan, R.; Bozzelli, J. W. Int. J. Quantum Chem. 2012, 112, 1945.

(c) Alvarez, L.; Bianco, C. L.; Toscano, J. P.; Lin, J.; Akaike, T.; Fukuto, J. M. Antioxid. Redox Signaling 2017, 27, 622.

[6] Ida, T.; Sawa, T.; Ihara, H.; Tsuchiya, Y.; Watanabe, Y.; Kumagai, Y.; Suematsu, M.; Motohashi, H.; Fujii, S.; Matsunaga, T.; Yamamoto, M.; Ono, K.; Devarie-Baez, N. O.; Xian, M.; Fukuto, J. M.; Akaike, T. Proc. Natl. Acad. Sci. U. S. A. 2014, 111, 7606.

[7] (a) Chen, X. L.; Jhee, K. H.; Kruger, W. D. J. Biol. Chem. 2004, 279, 52082 .

(b) Singh, S.; Padovani, D.; Leslie, R. A.; Chiku, T.; Banerjee, R. J. Biol. Chem. 2009, 284, 22457.

(c) Mishanina, A. V.; Libiad, M.; Banerjee, R. Nat. Chem. Biol. 2015, 11, 457.

[8] Akaike, T.; Ida, T.; Wei, F.-Y.; Nishida, M.; Kumagai, Y.; Alam, M. M.; Ihara, H.; Sawa, T.; Matsunaga, T.; Kasamatsu, S.; Nishimura, A.; Morita, M.; Tomizawa, K.; Nishimura, A.; Watanabe, S.; Inaba, K.; Shima, H.; Tanuma, N.; Jung, M.; Fujii, S.; Watanabe, Y.; Ohmuraya, M.; Nagy, P.; Feelisch, M.; Fukuto, J. M.; Motohashi, H. Nat. Commun. 2017, $8,1177$.

[9] (a) Toohey, J. I. Anal. Biochem. 2011, 413, 1

(b) Greiner, R.; Palinkas, Z.; Baesell, K.; Becher, D.; Antelmann, H.; Nagy, P.; Dick, T. P. Antioxid. Redox Signaling 2013, 19, 1749.

(c) Kimura, H. Free Radical Biol. Med. 2016, 96, S4.

(d) Yadav, P. K.; Martinov, M.; Vitvitsky, V.; Seravalli, J.; Wedmann, R.; Filipovic, M. R.; Banerjee, R. J. Am. Chem. Soc. 2016, 138, 289.

[10] (a) Hildebrandt, T. M.; Grieshaber, M. K. FEBS J. 2008, 275, 3352; (b) Libiad, M.; Yadav, P. K.; Vitvitsky, V.; Martinov, M.; Banerjee, R. J. Biol. Chem. 2014, 289, 30901.

(c) Landry, A. P.; Moon, S.; Kim, H.; Yadav, P. K.; Guha, A.; Cho, U.-S.; Banerjee, R. Cell Chem. Biol. 2019, 26, 1515.

[11] (a) Ihara, H.; Kasamatsu, S.; Kitamura, A.; Nishimura, A.; Tsutsuki, H.; Ida, T.; Ishizaki, K.; Toyama, T.; Yoshida, E.; Hamid, H. A.; Jung, M.; Matsunaga, T.; Fuji, S.; Sawa, T.; Nishida, M.; Kumagai, Y.; Akaike, T. Chem. Res. Toxicol. 2017, 30, 1673.

(b) Nishida, M.; Nishimura, A.; Matsunaga, T.; Motohashi, H.; Kasamatsu, S.; Akaike, T. Free Radical Biol. Med. 2017, 109, 132. (c) Khan, S.; Fujii, S.; Matsunaga, T.; Nishimura, A.; Ono, K.; Ida, T.; Ahmed, K. A.; Okamoto, T.; Tsutsuki, H.; Sawa, T.; Akaike, T. Cell Chem. Biol. 2018, 25, 1403.

(d) Kasamatsu, S. Antioxid. Redox Signaling 2020, 33, 1320.

(e) Kanda, H.; Kumagai, Y. Yakugaku Zasshi 2020, 140, 1119.

[12] (a) Sawa, T.; Zaki, M. H.; Okamoto, T.; Akuta, T.; Tokutomi, Y.; Kim-Mitsuyama, S.; Ihara, H.; Kobayashi, A.; Yamamoto, M.; Fujii, S.; Arimoto, H.; Akaike, T. Nat. Chem. Biol. 2007, 3, 727.

(b) Fujii, S.; Sawa, T.; Ihara, H.; Tong, K. I.; Ida, T.; Okamoto, T.; Ahtesham, A. K.; Ishima, Y.; Motohashi, H.; Yamamoto, M.; Akaike, T. J. Biol. Chem. 2010, 285, 23970.

(c) Fujii, S.; Sawa, T.; Nishida, M.; Ihara, H.; Ida, T.; Motohashi,
H.; Akaike, T. Arch. Biochem. Biophys. 2016, 595, 140.

[13] (a) Abiko, Y.; Yoshida, E.; Ishii, I.; Fukuto, J. M.; Akaike, T.; Kumagai, Y. Chem. Res. Toxicol. 2015, 28, 1301.

(b) Millikin, R.; Bianco, C. L.; White, C.; Saund, S. S.; Henriquez, S.; Sosa, V.; Akaike, T.; Kumagai, Y.; Soeda, S.; Toscano, J. P.; Lin, J.; Fukuto, J. M. Free Radical Biol. Med. 2016, 97, 136.

(c) Zivanovic, J.; Kouroussis, E.; Schott-Roux, S.; Filipovic, M. R. Free Radical Biol. Med. 2017, 112, 187.

(d) Ju, Y.; Fu, M.; Stokes, E.; Wu, L.; Yang, G. Molecules 2017, 22, 1334.

(e) Aroca, A.; Gotor, C.; Romero, L. C. Front. Plant Sci. 2018, 9, 1369.

(f) Kimura, H. Brit. J. Pharmacol. 2020, 177, 720.

(g) Pedre, B.; Dick, T. P. Biol. Chem. 2021, 402, 223

[14] (a) Bailey, T. S.; Zalcharov, L. N.; Pluth, M. D. J. Am. Chem. Soc. 2014, 136, 10573.

(b) Bailey, T. S.; Pluth, M. D. Free Radical Biol. Med. 2015, 89, 662.

[15] (a) Liang, D.; Wu, H.; Wong, M. W.; Huang, D. Org. Lett. 2015, 17, 4196.

(b) Cai, Y.-R.; Hu, C.-H. J. Phys. Chem. B 2017, 121, 6359.

(c) Cerda, M. M.; Hammers, M. D.; Earp, M. S.; Zakharov, L. N.; Pluth, M. D. Org. Lett. 2017, 19, 2314.

(d) Nagai, S.; Yoshida, M.; Takigawa, Y.; Torii, S.; Koshiishi, I. Food Chem. 2020, 128511.

[16] Zheng, Y.; Yu, B.; Li, Z.; Yuan, Z.; Organ, C. L.; Trivedi, R. K.; Wang, S.; Lefer, D. J.; Wang, B. Angew. Chem., Int. Ed. 2017, 56, 11749 .

[17] Yuan, Z.; Zheng, Y.; Yu, B.; Wang, S.; Yang, X.; Wang, B. Org. Lett. 2018, 20, 6364

[18] Jarosz, A. P.; Wei, W.; Gauld, J. W.; Auld, J.; Ozcan, F.; Aslan, M.; Mutus, B. Free Radical Biol. Med. 2015, 89, 512.

[19] Dillon, K. M.; Carrazzone, R. J.; Wang, Y.; Powell, C. R.; Matson, J. B. ACS Macro Lett. 2020, 9, 606 .

[20] Powell, C. R.; Dillon, K. M.; Wang, Y.; Carrazzone, R. J.; Matson, J. B. Angew. Chem., Int. Ed. 2018, 57, 6324.

[21] Kuivila, H. G. J. Am. Chem. Soc. 1954, 76, 870.

[22] Monks, T. J.; Jones, D. C. Curr. Drug Metab. 2002, 3, 425.

[23] Bora, P.; Chauhan, P.; Manna, S.; Chakrapani, H. Org. Lett. 2018, 20, 7916.

[24] Hankins, R. A.; Suarez, S. I.; Kalk, M. A.; Green, N. M.; Harty, M. N.; Lukesh, J. C. Angew. Chem., Int. Ed. 2020, 59, 22238.

[25] (a) Fridovich, I. J. Biol. Chem. 1997, 272, 18515.

(b) Bandyopadhyay, U.; Das, D.; Banerjee, R. K. Curr. Sci. 1999, 77,658

[26] Wang, Y.; Dillon, K. M.; Li, Z.; Winckler, E. W.; Matson, J. B. Angew. Chem., Int. Ed. 2020, 59, 16698.

[27] Roger, T.; Raynaud, F.; Bouillaud, F.; Ransy, C.; Simonet, S.; Crespo, C.; Bourguignon, M.; Villeneuve, N.; Vilaine, J.; Artaud, I. ChemBioChem 2013, 14, 2268.

[28] Artaud, I.; Galardon, E. ChemBioChem 2014, 15, 2361.

[29] Kang, J.; Xu, S.; Radford, M. N.; Zhang, W.; Kelly, S. S.; Day, J. J.; Xian, M. Angew. Chem., Int. Ed. 2018, 57, 5893.

[30] Khodade, V. S.; Toscano, J. P. J. Am. Chem. Soc. 2018, 140, 17333.

[31] Khodade, V. S.; Pharoah, B. M.; Paolocci, N.; Toscano, J. P. J. Am. Chem. Soc. 2020, 142, 4309.

[32] Chaudhuri, A.; Venkatesh, Y.; Das, J.; Gangopadhyay, M.; Maiti, T. K.; Singh, N. D. P. J. Org. Chem. 2019, 84, 11441.

[33] Fan, Y.; Pedersen, O. Nat. Rev. Microbiol. 2021, 19, 55.

[34] Dillon, K. M.; Morrison, H. A.; Powell, C. R.; Carrazzone, R. J.; Ringel-Scaia, V. M.; Winckler, E. W.; Council-Troche, R. M.; Allen, I. C.; Matson, J. B. Angew. Chem., Int. Ed. 2021, 60, 6061.

$(\mathrm{Lu}, \mathrm{Y}$. 